\title{
Photoacoustic-guided focused ultrasound for accurate visualization of brachytherapy seeds with the photoacoustic needle
}

\author{
Mithun Kuniyil Ajith Singh \\ Vinay Parameshwarappa \\ Ellen Hendriksen \\ Wiendelt Steenbergen \\ Srirang Manohar
}




\section{Photoacoustic-guided focused ultrasound for accurate visualization of brachytherapy seeds with the photoacoustic needle}

\author{
Mithun Kuniyil Ajith Singh, ${ }^{a}$ Vinay Parameshwarappa, ${ }^{a}$ \\ Ellen Hendriksen, ${ }^{\text {b }}$ Wiendelt Steenbergen, ${ }^{a}$ and \\ Srirang Manohar, ${ }^{\mathrm{a}, *}$ \\ aUniversity of Twente, P.O. Box 217, Biomedical Photonic Imaging \\ Group, Enschede 7500 AE, The Netherlands \\ ${ }^{b}$ Department of Radiation Oncology, Medisch Spectrum Twente, P.O. \\ Box 50000, Enschede 7512 KZ, The Netherlands
}

\begin{abstract}
An important problem in minimally invasive photoacoustic (PA) imaging of brachytherapy seeds is reflection artifacts caused by the high signal from the optical fiber/needle tip reflecting off the seed. The presence of these artifacts confounds interpretation of images. In this letter, we demonstrate a recently developed concept called photoacoustic-guided focused ultrasound (PAFUSion) for the first time in the context of interstitial illumination PA imaging to identify and remove reflection artifacts. In this method, ultrasound (US) from the transducer is focused on the region of the optical fiber/needle tip identified in a first step using PA imaging. The image developed from the US diverging from the focus zone at the tip region visualizes only the reflections from seeds and other acoustic inhomogeneities, allowing identification of the reflection artifacts of the first step. These artifacts can then be removed from the PA image. Using PAFUSion, we demonstrate reduction of reflection artifacts and thereby improved interstitial PA visualization of brachytherapy seeds in phantom and ex vivo measurements on porcine tissue. (- The Authors. Published by SPIE under a Creative Commons Attribution 3.0 Unported License. Distribution or reproduction of this work in whole or in part requires full attribution of the original publication, including its DOI. [DOI: 10.1117/1.JBO.21.12.120501]
\end{abstract}

Keywords: photoacoustics; ultrasound; reflection artifacts; photoacoustic needle; photoacoustic-guided focused ultrasound; brachytherapy.

Paper 160481LRR received Jul. 11, 2016; accepted for publication Nov. 14, 2016; published online Dec. 6, 2016.

Prostate cancer is one of the most common forms of cancer among men. ${ }^{1}$ Depending on the stage and severity of prostate cancer, several therapeutic options such as radiation therapy, hormone therapy, and radical prostatectomy are generally used. Brachytherapy is a targeted radiation therapy treatment which enables high-precision delivery of radiation doses to the tumor area in the prostate. In brachytherapy, the localized

*Address all correspondence to: Srirang Manohar, E-mail: s.manohar@utwente.nl tumor area instead of the whole prostate is targeted with defined radiation dose, using multiple (50 to 100) tiny metallic radioactive sources implanted inside the prostate. In brachytherapy, complications may arise when there is a deviation between the planned and delivered radiation dose. ${ }^{2}$ Potential reasons for this deviation are seed migration, prostate motion, edema, or implantation errors. ${ }^{2}$ Mismatch between planned and delivered radiation dose can be avoided to a large extent by accurate visualization of seeds during the implantation procedure.

Real-time seed visualization during the implantation procedure is currently performed using transrectal ultrasound imaging. However, seeds are often challenging to visualize using ultrasound (US) due to their small size, their similarity in acoustic impedance with microcalcifications, and due to the presence of characteristic acoustic artifacts such as shadowing and reverberations. ${ }^{3}$

In photoacoustic (PA) imaging, pulsed light absorbed by the target emits US by the thermoelastic mechanism. ${ }^{4}$ This US is detected using a US detector array to build up an image. PA imaging offers spectroscopic optical absorption contrast with US resolution. ${ }^{5}$ Owing to the high optical absorption of metal compared to tissue, PA imaging offers excellent contrast in imaging brachytherapy seeds. ${ }^{3,6}$ Thus, PA imaging can complement US imaging for better visualization of brachytherapy seeds. ${ }^{3,6,7}$

Considering the imaging depth required, the PA needle $e^{8,9}$ with interstitial light delivery via a cutting percutaneous needle is generating much interest in imaging brachytherapy seeds. ${ }^{7} \mathrm{~A}$ challenge in interstitial PA imaging of brachytherapy seeds is the presence of reflection artifacts caused by the high PA signal from the optical fiber/needle tip reflecting off the seeds as shown in Fig. 1(b). These artifacts may appear in the region of interest and can reduce the contrast and causes difficulty in image interpretation as reflection artifacts can be misinterpreted as seeds. ${ }^{7}$ It is important to identify and reduce reflection artifacts for accurate interstitial PA visualization of brachytherapy seeds.

We recently proposed a method called photoacoustic-guided focused ultrasound (PAFUSion) to identify reflection artifacts in epi-mode PA imaging. ${ }^{10}$ In this work, we study the use of PAFUSion in identifying and reducing in-plane reflection artifacts with the use of the PA needle in the visualization of brachytherapy seeds. We present the results of the combined approach in visualizing nonradioactive brachytherapy seeds in a tissue mimicking phantom and an ex vivo porcine tissue. In addition, we also show the potential of using multispectral PA for separating seed signals from other optical absorbers like blood in a phantom measurement.

The system consists of a commercial handheld US imager (MyLab_One, Esaote Europe BV, Maastricht, The Netherlands) with a linear array probe SL3323 (128 elements, $7.5 \mathrm{MHz}, 100 \%$ bandwidth). ${ }^{11}$ The system is used in the research mode with 64 elements where US transmission, laser pulse transmission, and data acquisition are controlled using custom-made software running on a PC. Illumination uses an Nd:YAG laser (Quanta-Ray Pro 250, Spectra Physics, Mountain View, California) pumping an optical parametric oscillator (VersaScan-L532, GWU, Erftstadt, Germany) at $10 \mathrm{~Hz}$. The energy per pulse is monitored using a photodetector. A multimode optical fiber (NA 0.22, diameter $600 \mu \mathrm{m}$ ) coupled to the laser delivers $10 \mathrm{~ns}$ laser light with $1.2 \mathrm{~mJ} /$ pulse at $800 \mathrm{~nm}$. For the multiwavelength studies, the range of 720 to $860 \mathrm{~nm}$ was covered with $5 \mathrm{~nm}$ steps; single wavelength studies used $800 \mathrm{~nm}$. The fiber is held in a biopsy needle $(14 \mathrm{G} \times 13 \mathrm{~cm}$, Bard Ltd., UK) and slide-fits into the $14 \mathrm{G}$ 


\section{JBO Letters}

(a)

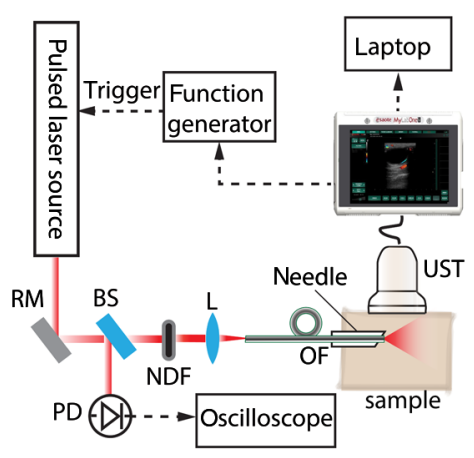

(b)

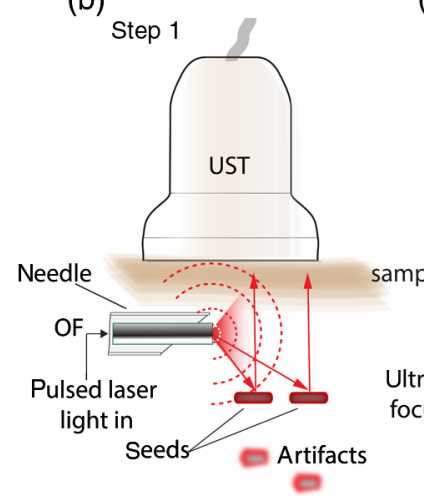

(c)

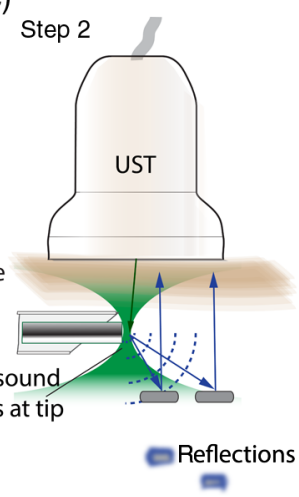

Fig. 1 (a) Block diagram of setup. RM, reflecting mirror; BS, glass plate to sample beam; PD, photodetector; NDF, neutral density filter; L, lens; OF, optical fiber; and UST, ultrasound transducer. (b) Concept of the PA needle, where pulsed light is applied in tissue via an optical fiber in a cutting needle with external US detection. By this, the tissue at the optical fiber tip, the tip itself, and the brachytherapy seeds can be visualized. Additionally, however, artifacts are also visualized due to reflection of the high PA signal from the fiber/needle tip on the seeds. (c) Concept of PAFUSion where in a second step; US from the transducer is focused at the optical fiber/needle tip. The US diverging from the focus zone at the tip is in a first approximation a mimic of the PA signal from tip in the first step. The detected signals visualize only the reflections from seed and other inhomogeneities allowing identification of the reflection artifacts of the first step.

cutting cannula [Fig. 1(a)]. The PA frame rate is $10 \mathrm{~Hz}$ and images can be displayed online using a GPU-based delay and sum reconstruction algorithm. Images presented here are averaged over 100 frames and reconstructed offline using a frequency domain reconstruction algorithm, ${ }^{12}$ assuming a constant speed of sound in the medium for all measurements (phantom measurement: $1480 \mathrm{~m} / \mathrm{s}$, ex vivo measurement: $1540 \mathrm{~m} / \mathrm{s}$ ).

The PAFUSion approach specifically in the context of the PA needle is shown in Figs. 1(b) and 1(c). Once the needle tip has been identified from the PA image (by manually identifying the position with the highest pixel intensity at the tip region in the image) in step 1 [Fig. 1(b)], US is focused to this point in a second step. The resultant US data processed using the PAFUSion algorithm ${ }^{10}$ generates an image with only reflections from deeper structures (PAFUSion image) hence representing the artifacts in the PA image from step 1 . The average image pixel value of the reflection artifact with the highest image value is then scaled to the image value of its counterpart in the PA image, and the same scaling is applied to all other identified artifacts in the PAFUSion image. After this, reconstructed and enveloped PAFUSion data are subtracted from the PA data and the positive parts of the result are plotted to obtain the corrected PA image. The negative parts of the result are assigned an image value of zero.
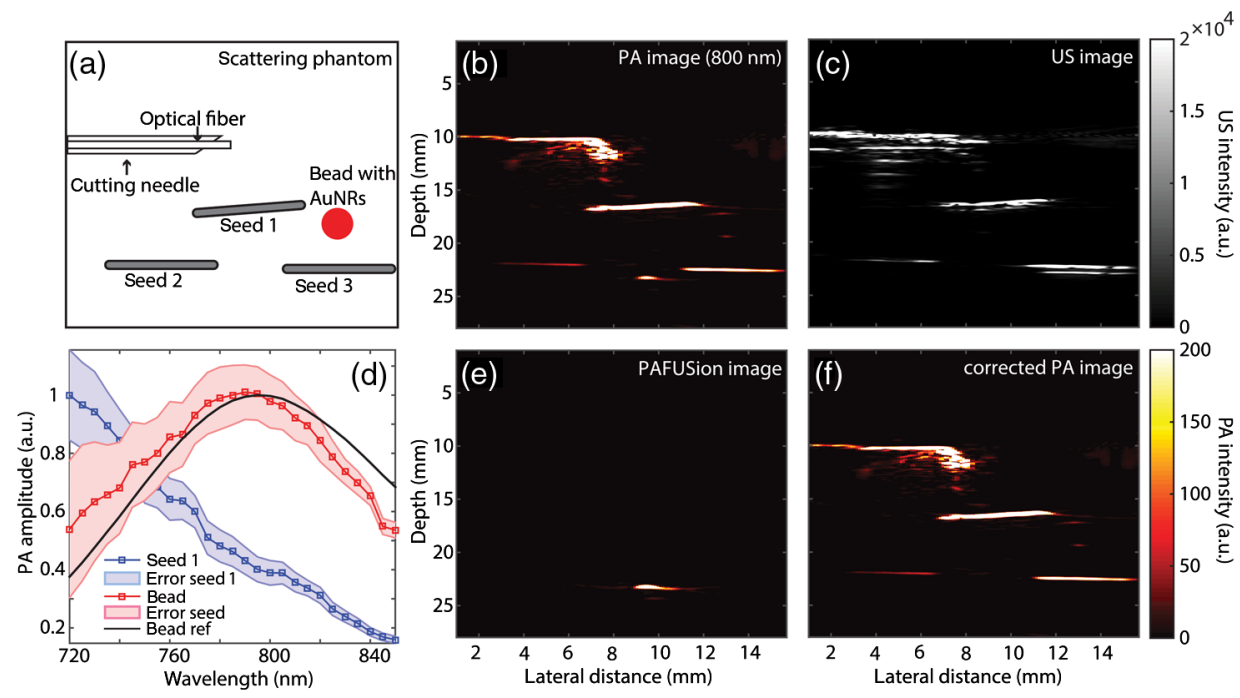

Fig. 2 (a) Schematic of the experiment showing orientation of the PA needle, arrangement of seeds and AuNR bead in the Agar phantom with Intralipid, (b) PA image at a wavelength of $800 \mathrm{~nm}$ showing the expected features and with an additional feature at $9.5 \times 24 \mathrm{~mm}^{2}$ (lateral distance $\times$ depth), (c) planewave US image, (d) measured PA spectrum of seed 1, bead with error margins (standard deviation of the averaged mean) compared with reference spectrum of the bead, (e) PAFUSion image showing that the unknown feature is a reflection artifact, and $(f)$ corrected PA image obtained by subtracting scaled PAFUSion image from the PA image. 
Figure 2(a) shows the schematic of the experiment to validate the proposed combined approach in a scattering phantom. The phantom $(10 \mathrm{~cm} \times 7.5 \mathrm{~cm} \times 5 \mathrm{~cm})$ was prepared with Agar dissolved in an aqueous solution of Intralipid (I141, 20\% emulsion, Sigma-Aldrich) to provide a bulk reduced scattering coefficient of $\mu_{\mathrm{s}}^{\prime}=1 \mathrm{~mm}^{-1}$. Within the phantom, three nonradioactive brachytherapy seeds (length $=4.5 \mathrm{~mm} \times 0.8 \mathrm{~mm}$, Bard Benelux N.V., The Netherlands) were embedded. The seeds were coated with a single layer of India ink $(\sim 200 \mu \mathrm{m})$ by dipping them in the ink and drying in air. India ink coating was used in this proof-of-principle study to improve the contrast of the brachytherapy seeds by around $10 \mathrm{~dB}$ in the PA image. For clinical implementation, a biocompatible coating with high optical absorption could be more suitable. A single bead (2.2-mm diameter) of sodium alginate carrying gold nanorods (AuNR) ${ }^{13}$ was also embedded in the phantom. The reference absorption spectrum of the bead [Fig. 2(d)], measured using a spectrophotometer (UV-2401PC, Shimadzu, Kyoto, Japan), shows a characteristic longitudinal plasmon absorption peak at $795 \mathrm{~nm}$. Water coupling was used in between the US probe and the phantom. The needle was oriented parallel to the US detector array. PA and plane-wave US images are acquired in the first step. Then after locating the fiber/needle tip, PAFUSion (focused US) data was also collected for offline processing. PA images for different excitation wavelengths ( 720 to $860 \mathrm{~nm}$ ) were also acquired.

Ex vivo validation of the approach was performed using four ink-coated brachytherapy seeds embedded inside porcine tissue. Using US guidance, the seeds were implanted inside the tissue using a standard 18G brachytherapy needle (Bard, Brachystar, Covington, Georgia) by one of the authors (E.M.). The same data acquisition and processing protocol were followed as for the phantom experiment. For both the phantom and ex vivo experiments, the seeds were aligned inside the imaging plane of the US transducer array.

Figure 2(b) shows the reconstructed PA image (phantom experiment) in which the shaft and the fiber/needle tip and all three seeds are visualized. The AuNR bead is seen only with top and bottom surfaces, as expected in a limited-view situation. The US image [Fig. 2(c)] shows the needle shaft and the seeds. In an ideal case, only the optically absorbing structures are expected to show up in the reconstructed PA image. However, a further feature is visible in the PA image (lateral distance $=9.5 \mathrm{~mm}$, depth $=24 \mathrm{~mm}$ ) which could be interpreted as an optically absorbing feature, e.g., another seed.

The PAFUSion image [Fig. 2(e)] reveals this feature as a reflection artifact. Figure 2(f) shows the corrected PA image obtained by subtracting the PAFUSion-identified reflection artifacts from the real PA image showing a reduction of the artifact. The image values of real PA features are unaffected in the corrected PA image. In Fig. 2(d), the PA image values of seed 1 and that of the AuNR bead (normalized to the measured pulse energy) are plotted against the wavelength, together with the reference spectrum of AuNR. The image values of ink-coated seeds decrease monotonically with wavelength, while the PA spectrum of the bead shows the signature longitudinal plasmon peak at roughly $795 \mathrm{~nm}$.

Orientation of the needle and arrangement of seeds inside the porcine tissue are schematically shown in Fig. 3(a). For better acoustic coupling, the sample was covered with transparent kitchen cling film $(\sim 20 \mu \mathrm{m})$ after smearing with US gel [Fig. 3(d)]. Figure 3(b) shows the reconstructed PA image in which PA needle/ fiber tip, all four seeds, and several other bright features are visible. It is difficult to interpret this image since several bright features with shapes similar to the seeds are present. The shaft of the PA needle, all the four seeds, and several other echogenic tissue structures are visible in the US image [Fig. 3(c)].

Figure 3(e) shows the PAFUSion image from which reflection artifacts in the PA image can be identified. The PAFUSion image shows that a number of features in the PA image are reflection artifacts. All of them are caused by the fiber/needle tip PA signal reflecting off the seeds and off other tissue echogenic structures. Figure 3(f) shows the corrected PA image obtained by subtracting the PAFUSion-identified reflection artifacts from the real PA image. It is evident that the reflection artifacts are significantly reduced by using PAFUSion without affecting the contrast of real PA features. An interesting additional feature is visible at the same depth of seed 3 in the PA image (lateral distance $=7.5 \mathrm{~mm}$, depth $=15 \mathrm{~mm}$ ). The
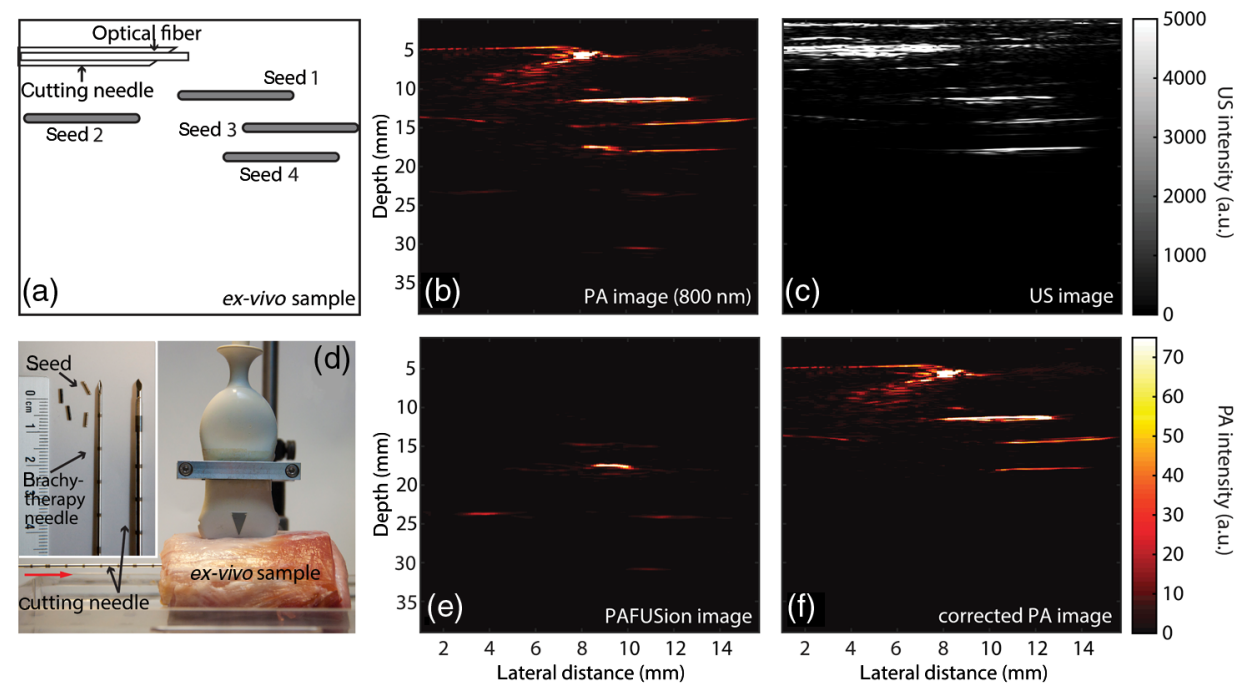

Fig. 3 (a) Schematic showing orientation of the PA needle and arrangement of seeds inside the porcine tissue, (b) PA image at a wavelength of $800 \mathrm{~nm}$, (c) plane-wave US image, (d) photograph of the imaging situation with needle inserted in the ex vivo sample. Inset is a photograph of the inner and outer needles alongside the seed. (e) PAFUSion image with the identified reflection artifacts, and (f) corrected PA image obtained by subtracting the scaled PAFUSion image from the PA image. 
PAFUSion image uncovered this feature as a reflection artifact. This would have been caused by the fiber/needle tip signal reflecting off the point-like tissue feature visible just above seed 1 (lateral distance $=7.5 \mathrm{~mm}$, depth $=10 \mathrm{~mm}$ ) in both the PA and US images.

Our approach has been successfully demonstrated in phantom and in ex vivo settings. The method has been developed in the context of PA needle imaging of brachytherapy seeds, but is eminently suited for all applications of the PA needle where reflection artifacts could result due to the presence of acoustic reflectors. However, the approach needs to be further investigated. One issue is that high absorption of heterogeneous tissue proximal to the needle tip could result in multiple bright PA features near the tip. This may create uncertainties in accurately identifying the fiber/needle tip which is essential for using the approach. Another issue that requires investigation is the approach used for correction of the PA images. Subtraction of the PAFUSion images from the PA image worked well in the situations studied, but will require optimization in the case of real PA features mixed with any reflection artifacts.

In addition, PAFUSion will be able to identify and reduce reflection artifacts generated inside the imaging plane only. If the fiber/needle tip is outside the imaging plane, it will be impossible to reduce reflection artifacts using our approach. However, in-plane needle insertion is commonly used in clinical practice for accurate guidance of the needle tip, making this less a limitation.

In linear US array-based PA imaging, one can expect significant contributions from absorbing objects outside the imaging plane as direct clutter. ${ }^{14}$ These out-of-plane signals may even reflect off echogenic structures inside the imaging plane to generate indirect echo clutter. ${ }^{14}$ However, these out-of-plane PA signals will be significant only when the tissue is illuminated from the outside in a noninvasive setting unlike the present interstitial illumination setting.

Angular distribution of ultrasonic wavefield after the focus depth is an important parameter when using PAFUSion in the context of the PA needle, as this angle defines the fieldof-view of the method in identifying and eliminating reflection artifacts. In our case, where the center frequency was $7.5 \mathrm{MHz}$ and the pitch was $0.245 \mathrm{~mm}$, this angle limit was at $\pm 24 \mathrm{deg}$, and was sufficient to identify the reflection artifacts in this study.

In both the phantom and ex vivo experiments, light energy was $1.2 \mathrm{~mJ} /$ pulse, resulting in a fluence of $425 \mathrm{~mJ} / \mathrm{cm}^{2}$ at the fiber tip. The maximum permissible exposure (MPE) for NIR light inside tissue is not defined (for skin the MPE is $20 \mathrm{~mJ} / \mathrm{cm}^{2}$ for the wavelength used). However, the high exposure at the tip raises safety concerns from a clinical standpoint. We envisage addressing this issue in future using fiber tip modifications, e.g., using a diffusing tip. ${ }^{15}$

Coating the needle tip with a dye/paint with a spectral signature and identifying the reflection artifacts caused by it using spectral decomposition and correcting for it will potentially be an alternate solution for the reflection artifact reduction in interstitial PA imaging. However, this requires further investigation.

We conclude that PAFUSion enables identification and substantial reduction of reflection artifacts when using the PA needle, considerably improving the visualization of brachytherapy seeds. Our phantom results also confirm that multispectral $\mathrm{PA}^{6}$ can be potentially a useful tool to separate signals from the brachytherapy seeds and other optical absorbers in the tissue. The
PAFUSion approach reported here is suitable for real-time implementation since it requires only a single focus US measurement and an image subtraction step. In a future clinical scenario, we envisage that the PA needle in combination with realtime PAFUSion will provide accurate visualization of the distribution of brachytherapy seeds, which in turn provide feedback to the radiotherapist regarding adherence to the therapy plan.

\section{Disclosures}

Authors declare that there is no conflict of interest regarding the publication of this paper.

\section{Acknowledgments}

M.K.A.S. and W.S. are funded by the European Community's Seventh Framework Programme (FP7/2007-2013) under grant Agreement No. 318067. S.M. is funded by the PIHC-Pioneers in Healthcare Innovation funds 2014, and the FP7 program INDIGO Policy in project RTQ-PAI. Authors acknowledge Dr. Altaf Hussain and Johan van Hespen for their insightful discussions and help in experiments. Dr. Peter Brands and engineers from ESAOTE Europe BV are thanked for discussions and support with the MyLab_One system.

\section{References}

1. J. Crook, "The role of brachytherapy in the definitive management of prostate cancer," Cancer Radiother. 15(3), 230-237 (2011).

2. E. M. Messing et al., "Intraoperative optimized inverse planning for prostate brachytherapy: early experience," Int. J. Radiat. Oncol. Biol. Phys. 44(4), 801-808 (1999).

3. J. L. Su et al., "Photoacoustic imaging of prostate brachytherapy seeds," Biomed. Opt. Express 2(8), 2243-2254 (2011).

4. Y. Zhou, J. Yao, and L. V. Wang, "Tutorial on photoacoustic tomography," J. Biomed. Opt. 21(6), 061007 (2016).

5. M. Kuniyil Ajith Singh, W. Steenbergen, and S. Manohar, "Handheld probe-based dual mode ultrasound/photoacoustics for biomedical imaging," in Frontiers in Biophotonics for Translational Medicine, M. Olivo and U. S. Dinish, Eds., pp. 209-247, Springer, Singapore (2016).

6. T. Harrison and R. J. Zemp, "Coregistered photoacoustic-ultrasound imaging applied to brachytherapy," J. Biomed. Opt. 16(8), 080502 (2011).

7. M. A. L. Bell et al., "In vivo visualization of prostate brachytherapy seeds with photoacoustic imaging," J. Biomed. Opt. 19(12), 126011 (2014).

8. D. Piras et al., "Photoacoustic needle: minimally invasive guidance to biopsy," J. Biomed. Opt. 18(7), 070502 (2013).

9. W. Xia et al., "Performance characteristics of an interventional multispectral photoacoustic imaging system for guiding minimally invasive procedures," J. Biomed. Opt. 20(8), 086005 (2015).

10. M. Kuniyil Ajith Singh and W. Steenbergen, "Photoacoustic-guided focused ultrasound (PAFUSion) for identifying reflection artifacts in photoacoustic imaging," Photoacoustics 3(4), 123-131 (2015).

11. K. Daoudi et al., "Handheld probe integrating laser diode and ultrasound transducer array for ultrasound/photoacoustic dual modality imaging," Opt. Express 22(21), 26365-26374 (2014).

12. M. Jaeger et al., "Fourier reconstruction in optoacoustic imaging using truncated regularized inverse k-space interpolation," Inverse Probl. 23(6), S51-S63 (2007).

13. R. G. Rayavarapu et al., "Synthesis and bioconjugation of gold nanoparticles as potential molecular probes for light-based imaging techniques," Int. J. Biomed. Imaging 2007, 29817 (2007).

14. M. Jaeger, J. C. Bamber, and M. Frenz, "Clutter elimination for deep clinical optoacoustic imaging using localised vibration tagging (LOVIT)," Photoacoustics 1(2), 19-29 (2013).

15. T. Mitcham et al., "Photoacoustic imaging driven by an interstitial irradiation source," Photoacoustics 3(2), 45-54 (2015). 